\title{
四丁基碘化胺催化活化烯烃烷氧羰基化反应：合成异喹啉二酮衍生物
}

\author{
唐裕才张敏李小青许响生杜晓华* \\ (浙江工业大学化学工程学院 杭州 310014)
}

\begin{abstract}
摘要 异喹啉二酮骨架是一种重要的有机合成砌块, 广泛存在于生物碱、天然产物和药物中. 提供了一种非金属催化 构建异喹啉二酮骨架结构的新方法. 机理研究表明, 该反应经历了一个自由基过程: 在四丁基碘化胺催化作用下, 肼 化合物质子被逐步擭取生成烷氧羰基自由基，随后对活化烯烃进行自由基加成反应，进一步环化形成异哇啉二酮骨架. 该方法具有反应条件温和、底物普适性广、环境友好等特点, 为合成含异喹啉二酮骨架分子结构提供了一种新途径. 关键词＼cjkstart异喹啉二酮结构; 非金属催化; 四丁基碘化胺; 自由基加成/环化
\end{abstract}

\section{Tetrabutylammonium lodide-Catalyzed Radical Alkoxycarbonylation of Activated Alkenes: Synthesis of Isoquinolinedione Derivatives}

\author{
Tang, Yucai Zhang, Min Li, Xiaoqing Xu, Xiangsheng Du, Xiaohua* \\ (College of Chemical Engineering, Zhejiang University of Technology, Hangzhou 310014)
}

\begin{abstract}
The isoquinolinedione skeletons are important building blocks, which have been widely present in plant alkaloids, natural products and pharmaceuticals. In this paper, a novel method to construct isoquinolinedione framework was developed, which can be easily operated under metal-free conditions. In the presence of tetrabutylammonium-iodide catalyst, hydrogen atoms were abstracted step by step from carbazate to generate methoxycarbonyl radical which followed by radical addition/ cyclization reaction leading to isoquinolinedione. The presented methodology will provide new strategies for construct isoquinolinedione-type products, which has advantages of simple operation, mild reaction conditions, and environmentally benign.
\end{abstract}

Keywords isoquinolinedione skeleton; metal-free; tetrabutylammonium-iodide; radical addition/cyclization

异喹啉二酮骨架结构是一种重要的有机结构基础 单元，具有独特的生物活性及广泛的合成应用价值，大 量存在于天然产物和重要的药物分子中 ${ }^{[1 \sim 3]}$. 如有生物 活性的醛糖还原酶抑制剂、选择性细胞周期蛋白激酶抑 制剂、酪氨酸激酶抑制剂等(图 1). 因此, 发展一种快速 高效地合成这种骨架结构的新方法显得尤为迫切.

目前，文献报道合成异喹啉二酮骨架结构的方法主 要包括: (1) 2002 年, Zard 课题组 ${ }^{[4]}$ 提出了一种黄原酸酯 法构建异喹啉二酮结构. 该方法中, 黄原酸酯在高温下 经过自由基引发剂形成一个碳自由基, 随后对芳环进攻 环化形成这种结构(图 2). (2)今年刘群课题组 ${ }^{[5]}$ 提出了 另外一种新方法, 在碱催化下, $\alpha, \beta$-不饱和羰基化合物 和二烷基戊烯二酸一锅法经历 $[3+3]$ 环化/分子内氮环
化得到最终产物(图 2). 这些方法对于合成异喹啉二酮 骨架结构具有十分重要的参考价值. 近年来, 自由基反 应引起了化学家的广泛关注, 尤其是自由基对活化烯烃 进行自由基加成反应构建杂环化合物的研究取得了较 大的进展 ${ }^{[6,7]}$. 我们课题组研究发现, 在四丁基碘化胺/ 叔丁基过氧化氢(TBAI-TBHP)催化氧化下，磺酰基肼化 合物被逐步㩲取质子形成磺酰基自由基，随后该自由基 对烯烃进行自由基加成反应形成 $\mathrm{C}-\mathrm{S}$ 键, 实现了烯烃 的直接磺酰化反应 ${ }^{[8 \mathrm{a} \sim 8 c]}$. 最近, 我们课题组 ${ }^{[8 \mathrm{~d}]}$ 报道了以 肼基甲酸甲酯和 $N$-芳基取代的丙烯酰胺类化合物为原 料通过自由基加成/关环反应合成了一系列含有酯基的 吲哚-2-酮类化合物. 该反应的突出特点是利用廉价的 铁作为催化剂，在温和条件下引入酯基官能团，副产物

*E-mail: duxiaohua@zjut.edu.cn

Received October 11, 2014; revised November 19, 2014; published online December 2, 2014

Project supported by the National Natural Science Foundation of China (No. 21102130) and the Key Innovation Team of Science \& Technology in Zhejiang Province (Nos. 2010R50018, 2011R50017).

国家自然科学基金(No. 21102130)和浙江省科技创新团队(Nos. 2010R50018, 2011R50017)资助项目. 


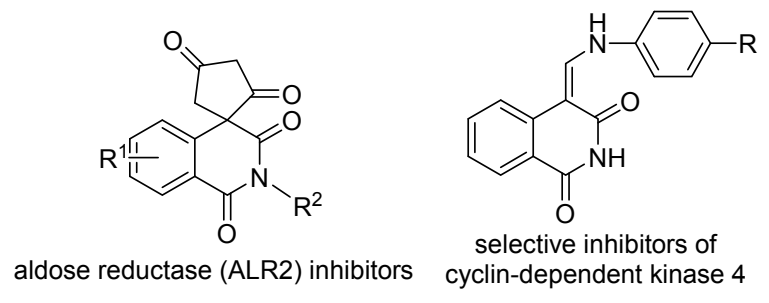<smiles>[R1]Nc1nc2ccc3c(c2[nH]1)C(=O)NC(=O)C3(C)C</smiles>

inhibitors of

Lck kinase

图 1 具有生物活性的异喹啉二酮骨架衍生物

Figure 1 Biologically active isoquinolinedione derivatives

是无毒的氮气, 因此是一个环境友好的化学反应. 我们 小组在自己 ${ }^{[8]}$ 及其他小组 ${ }^{[9]}$ 工作的基础上, 继续探究了 肼基甲酸甲酯作为自由基来源的适用范围, 在这里报道 一些近期所获得的研究结果(图 2).

\section{1 结果与讨论}

\section{1 最佳反应条件的选择}

以 $N$-丙烯酰基- $N$-甲基-3-硝基苯甲酰胺和肼甲酸甲 酯作为标准底物进行实验条件优化, 考察了催化剂、氧 化剂、溶剂和温度对该反应的影响, 实验结果见表 1 . 从 表 1 的数据可知, 在无催化剂的时候, 反应无法进行, 未检测到所需的产物(表 1, Entry 1). 对催化剂进行了进 一步的篎选, 如 $\mathrm{FeCl}_{2} \cdot 4 \mathrm{H}_{2} \mathrm{O} 、 \mathrm{TBAI} 、 \mathrm{KI} 、 \mathrm{FeSO}_{4} \cdot 7 \mathrm{H}_{2} \mathrm{O}$, 反 应都能得到中等收率的产物(表 1, Entries 2 5), 研究发 现其他的铜催化剂, 如 $\mathrm{CuBr}$ 和 $\mathrm{Cu}(\mathrm{OAc})_{2}$, 对该反应没 有催化效果或者得不到所需产物(表 1, Entries 6 7). 从
以上实验结果表明 TBAI 催化效果最佳(表 1, Entry 3). 以 TBAI 为催化剂, 接下来考察了其他的氧化剂, 如二 叔丁基过氧化物(DTBP)和过氧化苯甲酸叔丁酯(TBPB) 对该反应的影响, 研究结果表明改变氧化剂对该反应没 有效果，基本检测不到产物(表 1, Entries 8～9). 随后又 对常见的溶剂进行了笁选, 如 $\mathrm{H}_{2} \mathrm{O} 、 \mathrm{DMF} 、 \mathrm{EtOAc}$, 然 而反应产率明显下降(表 1 , Entries 10１2). 进一步又研 究了温度效应对反应的影响. 当将反应温度降低到 $60{ }^{\circ} \mathrm{C}$ 时, 反应的产率略微降低至 48\%(表 1, Entry 13). 而当升高反应温度至 $100{ }^{\circ} \mathrm{C}$, 反应的产率上升至 67\%(表 1, Entry 14)，说明温度对反应有一定的影响，然 而继续升温至 $120{ }^{\circ} \mathrm{C}$, 反应收率又下降至 54\%(表 1 , Entry 15). 在考察了各方面的因素后, 确定了该反应的 最佳反应条件为: 以 TBAI 为催化剂, TBHP 为氧化剂, 在乙腈中 $100{ }^{\circ} \mathrm{C}$ 下反应.

\section{2 反应适用性研究}

在最佳反应条件下，对该反应体系的底物普适性进 行了考察, 一系列 $N$-丙烯酰基- $N$-甲基苯甲酰胺衍生物 都能应用于该体系, 如表 2 所示. 改变氮原子上的保护 基团，如甲基、乙基、苄基和苯基，反应都能较好的进 行下去,以中等收率得到产物, 其中当保护基团为苯基 和茮基时，反应活性比脂肪族族高，分别以 $50 \%$ 和 $42 \%$ 收率得到产物(表 2, Entries 3 6). 随后对一系列不同芳 环取代的反应底物进行了研究. 当取代基在苯环对位上 时, 不论是供电子基(如甲基、乙氧基和叔丁基)还是吸 电子基(如卤素、三氟甲基、酯基)，反应都能顺利进行， 产率无明显差异(表 2, Entries 7 14). 当取代基在苯环 间位时, 强吸电子基团硝基表现出较好的反应活性(表 2, Entries 15 17). 而当取代基在邻位时，如甲基、碘、

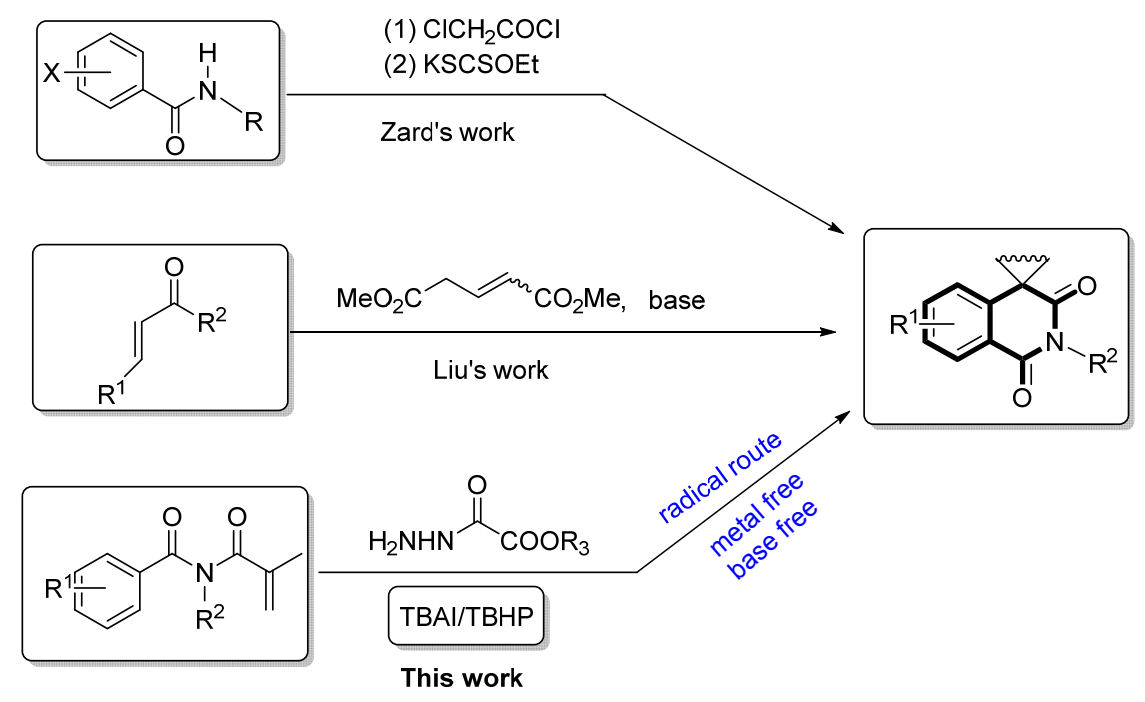

图 2 不同构建异喹啉二酮骨架结构的方法

Figure 2 Different methods for isoquinolinedione skeleton 
表 1 反应条件优化 ${ }^{a}$

Table 1 Reaction conditions optimization

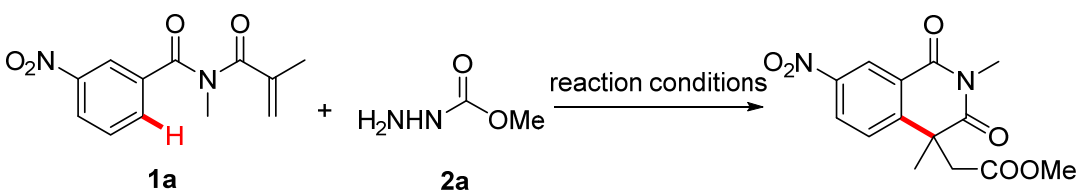

\begin{tabular}{|c|c|c|c|c|c|}
\hline Entry & Cat. & Solvent & $t /{ }^{\circ} \mathrm{C}$ & Oxidant & Yield $^{b} / \%$ \\
\hline 1 & - & $\mathrm{MeCN}$ & 80 & ТВHP & 0 \\
\hline 2 & $\mathrm{FeCl}_{2} \bullet 4 \mathrm{H}_{2} \mathrm{O}$ & $\mathrm{MeCN}$ & 80 & ТВНP & 42 \\
\hline 3 & TBAI & $\mathrm{MeCN}$ & 80 & ТВНP & 59 \\
\hline 4 & $\mathrm{KI}$ & $\mathrm{MeCN}$ & 80 & ТВHP & 53 \\
\hline 5 & $\mathrm{FeSO}_{4} \cdot 7 \mathrm{H}_{2} \mathrm{O}$ & $\mathrm{MeCN}$ & 80 & ТВНР & 46 \\
\hline 6 & $\mathrm{CuBr}$ & $\mathrm{MeCN}$ & 80 & ТВHР & Trace \\
\hline 7 & $\mathrm{Cu}(\mathrm{OAc})_{2}$ & $\mathrm{MeCN}$ & 80 & ТВHP & 0 \\
\hline 8 & TBAI & $\mathrm{MeCN}$ & 80 & DTBP & N.R \\
\hline 9 & TBAI & $\mathrm{MeCN}$ & 80 & ТВРВ & Trace \\
\hline 10 & TBAI & $\mathrm{H}_{2} \mathrm{O}$ & 80 & ТВНP & 14 \\
\hline 11 & TBAI & $\mathrm{DMF}$ & 80 & ТВHP & 26 \\
\hline 12 & TBAI & EtOAc & 80 & ТВHP & 40 \\
\hline 13 & TBAI & $\mathrm{MeCN}$ & 60 & ТВНР & 48 \\
\hline 14 & TBAI & $\mathrm{MeCN}$ & 100 & ТВHP & 67 \\
\hline 15 & TBAI & $\mathrm{MeCN}$ & 120 & TBHP & 54 \\
\hline
\end{tabular}

${ }^{a}$ Reaction conditions: 1a $(0.25 \mathrm{mmol})$, 2a $(1.5 \mathrm{mmol})$, catalyst $(20 \mathrm{mmol} \%)$ and TBHP $\left(1.5 \mathrm{mmol}, 70 \%\right.$. aqueous solution) in solvent $(2.0 \mathrm{~mL})$ for $8 \mathrm{~h} ;{ }^{b}$ isolated yield.

表 2 反应范围 ${ }^{a}$

Table 2 The reaction scope

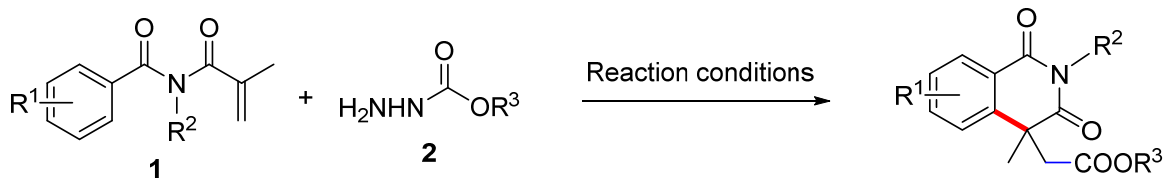

\begin{tabular}{|c|c|c|c|c|}
\hline Product & $\mathrm{R}^{1}$ & $\mathrm{R}^{2}$ & $\mathrm{R}^{3}$ & Yield $^{b} / \%$ \\
\hline 3 & $\mathrm{H}$ & $\mathrm{Me}$ & $\mathrm{Me}$ & 36 \\
\hline 4 & $\mathrm{H}$ & $\mathrm{Bn}$ & $\mathrm{Me}$ & 50 \\
\hline 5 & $\mathrm{H}$ & Et & $\mathrm{Me}$ & 23 \\
\hline 6 & $\mathrm{H}$ & $\mathrm{Ph}$ & $\mathrm{Me}$ & 42 \\
\hline 7 & 4-Me & $\mathrm{Me}$ & $\mathrm{Me}$ & 67 \\
\hline 8 & $4-\mathrm{C}_{2} \mathrm{H}_{5} \mathrm{O}$ & $\mathrm{Me}$ & $\mathrm{Me}$ & 63 \\
\hline 9 & $4-\mathrm{F}$ & $\mathrm{Me}$ & $\mathrm{Me}$ & 68 \\
\hline 10 & 4-Cl & $\mathrm{Me}$ & $\mathrm{Me}$ & 70 \\
\hline 11 & $4-\mathrm{Br}$ & $\mathrm{Me}$ & $\mathrm{Me}$ & 57 \\
\hline 12 & $4-\mathrm{CF}_{3}$ & $\mathrm{Me}$ & $\mathrm{Me}$ & 61 \\
\hline 13 & $4-\mathrm{CO}_{2} \mathrm{Me}$ & $\mathrm{Me}$ & $\mathrm{Me}$ & 59 \\
\hline 14 & $4-\mathrm{C}\left(\mathrm{CH}_{3}\right)_{3}$ & $\mathrm{Me}$ & $\mathrm{Me}$ & 60 \\
\hline 15 & $3-\mathrm{NO}_{2}$ & $\mathrm{Me}$ & $\mathrm{Me}$ & 67 \\
\hline 16 & $3-\mathrm{CH}_{3}$ & $\mathrm{Me}$ & $\mathrm{Me}$ & 55 \\
\hline 17 & $3-I$ & $\mathrm{Me}$ & $\mathrm{Me}$ & 39 \\
\hline 18 & $2-\mathrm{CH}_{3}$ & $\mathrm{Me}$ & $\mathrm{Me}$ & 47 \\
\hline $19^{c}$ & $2-\mathrm{I}$ & $\mathrm{Me}$ & $\mathrm{Me}$ & 35 \\
\hline 20 & $3,4-(\mathrm{MeO})_{2}$ & $\mathrm{Me}$ & $\mathrm{Me}$ & 44 \\
\hline 21 & $3,5-(\mathrm{MeO})_{2}$ & $\mathrm{Me}$ & $\mathrm{Me}$ & 48 \\
\hline 22 & $\mathrm{H}$ & $\mathrm{Me}$ & Et & 51 \\
\hline
\end{tabular}

${ }^{a}$ Reaction conditions: $1(0.25 \mathrm{mmol}), \mathbf{2}(1.5 \mathrm{mmol}), \mathrm{TBAI}(20 \mathrm{~mol} \%)$, TBHP $\left(1.5 \mathrm{mmol}, 70 \%\right.$. aqueous solution) in $\mathrm{MeCN}(2.0 \mathrm{~mL})$ at $100{ }^{\circ} \mathrm{C}$ for $8 \mathrm{~h}$; ${ }^{b}$ isolated yield; ${ }^{c}$ reaction time is $12 \mathrm{~h}$. 
可能是由于空间位阻的原因, 导致反应收率相对较低 (表 2, Entries 18 19). 最后考察了苯环多取代对该反应 的影响, 无论是 3,4 位取代还是 3,5 位取代, 对该反应没 有太大的影响, 产率无明显差异(表 2, Entries 20２1). 其他的肼化合物, 如肼甲酸乙酯也能较好的兼容该反 应，以 51\%的收率得到最终产物(表 2, Entry 22).

\section{3 可能的反应机理}

为了探究反应的可能机理, 当加入当量的自由基捕 捉剂(TEMPO)时，实验发现该反应被抑制，检测不到产 物. 基于以上的实验结果并参考作者以前的工作 ${ }^{[8]}$, 推 测该反应可能经历了一个自由基过程. 首先, 在碘离子 催化下, 叔丁基过氧化氢产生叔丁基自由基或叔丁过氧 自由基，随后自由基对肼化合物逐步瞳取质子，脱去氮 气形成烷氧羰基自由基, 进一步对反应底物进行自由基 加成反应，形成中间体 $\mathbf{I}$, 紧接着该自由基对苯环进攻, 自由基环化形成中间体 II, 最后被叔丁基过氧化氢夺取 电子, 形成产物(Scheme 1).

\section{2 结论}

本文提供了一种简单有效的构建异喹啉二酮骨架 结构的新方法, 该方法中采用廉价的四丁基碘化胺/叔 丁基过氧化氢为催化氧化体系, 以较好的收率得到最终 产物, 该反应具有操作简便、底物适用范围广以及反应 条件温和等特点, 对于丰富杂环化合物的研究具有重要 的意义.

\section{3 实验部分}

\section{1 仪器与试剂}

Bruker AC-500 型核磁共振仪, TMS 为内标, $\mathrm{CDCl}_{3}$ 为溶剂; Bruker Esquire Hct 质谱仪, ESI 源(电喷雾离子 源); SB-1100 型旋转蒸发器(上海爱朗仪器有限公司); DLSB-10/40 型低温冷却液循环洜(巩义市予华仪器有限 责任公司); RY-1 型毛细管显微熔点仪, 天津分析仪器 厂，温度计未校正; 薄层色谱(TLC, Thin Layer Chromatography). 所用硅胶为 GF254, 青岛海洋化工厂产品, 紫外 $254 \mathrm{~nm}$ 或 $365 \mathrm{~nm}$ 观察. 所有溶剂和试剂均为分析 纯.

\section{2 实验方法}

3.2.1 $N$-丙烯酰基- $N$-甲基苯甲酰胺类化合物的合成 参考文献[9a]，取 $1.22 \mathrm{~g}(10 \mathrm{~mol})$ 苯甲酸和 $1.26 \mathrm{~g}$ (12 mol)草酰氯溶于 $15 \mathrm{~mL}$ 二氯甲烷中, 同时滴加 3 滴 $N, N$-二甲基甲酰胺(DMF)搅拌均匀,在 $0{ }^{\circ} \mathrm{C}$ 下搅拌反应, $2 \mathrm{~h}$ 后慢慢升至室温, 旋去溶剂, 粗产物无需分离直接 用于下一步反应.

将甲胺盐酸盐 $(20 \mathrm{~mol})$, 三乙胺 $(20 \mathrm{~mol})$ 溶于二氯甲 烷中, 待其充分溶解后, 慢慢滴加上述所得粗产物(二 氯甲烷作为溶剂), TLC 检测反应结束后, 加入 $5 \mathrm{~mL}$ 饱 和碳酸钠溶液，二氯甲烷萃取，合并有机相，无水硫酸 钠干燥, 旋去溶剂, 得粗产物, 无需纯化直接用于下一 步反应。
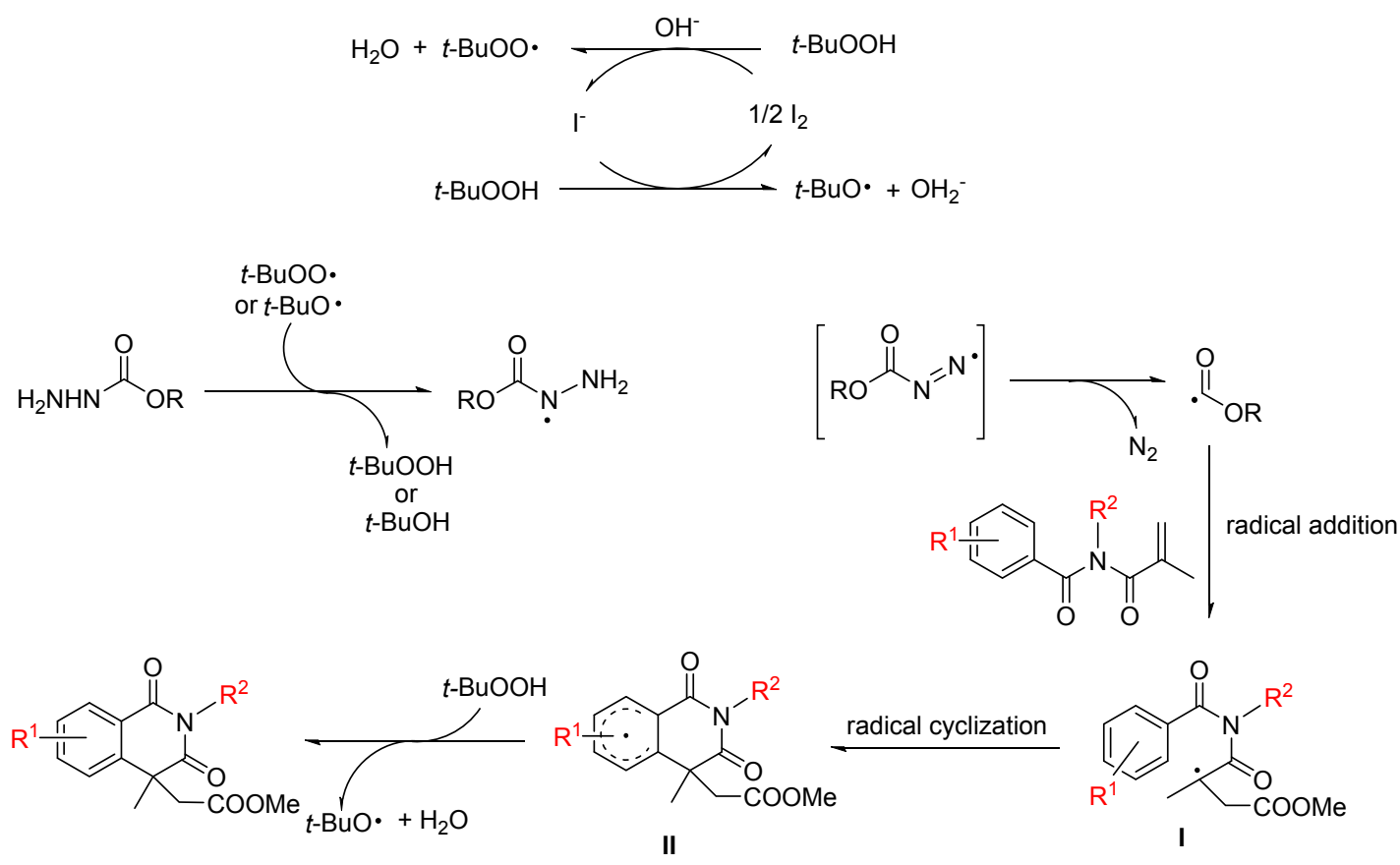

图式 1 可能反应机理

Scheme 1 Possible reaction mechanism 
将上述中所得产物 $(5 \mathrm{~mol})$ 、三乙胺 $(10 \mathrm{~mol})$ 和 DMAP (0.5 mol)溶于甲苯中, 慢慢滴加甲基丙烯酰氯 $(10 \mathrm{~mol})$, 滴加完毕后加热回流 $12 \mathrm{~h}, \mathrm{TLC}$ 检测反应结 束, 加入 $10 \mathrm{~mL}$ 饱和碳酸钠溶液, 二氯甲烷萃取, 合并 有机相, 无水硫酸镁干燥, 过滤, 旋蒸除去溶剂, 柱层 析分离得产物 $N$-丙烯酰基- $N$-甲基苯甲酰胺.

\subsection{2 目标物异喹啉二酮衍生物的合成}

将取代 $N$-丙烯酰基- $N$-甲基苯甲酰胺 $(0.25 \mathrm{mmol}$ )、 四丁基碘化胺 $(0.05 \mathrm{mmol})$ 与肼甲酸甲酯 $(1.5 \mathrm{mmol}$ )溶于 $2 \mathrm{~mL}$ 乙腈中, 加入叔丁基过氧化氢 $(1.5 \mathrm{mmol}, 70 \%$ 水溶 液), 在 $100{ }^{\circ} \mathrm{C}$ 下摚拌反应, TLC 检测反应结束后, 减压 旋去溶剂, 混合物再经柱层析分离提纯得目标产物 3 21.

2-(2,4-二甲基-1,3-二酮)-1,2,3,4-四氢异喹啉-4-乙酸 甲酯(3): 白色固体, 产率 $36 \%$. m.p. $102 \sim 103{ }^{\circ} \mathrm{C} ;{ }^{1} \mathrm{H}$ $\operatorname{NMR}\left(500 \mathrm{MHz}, \mathrm{CDCl}_{3}\right) \delta: 8.28(\mathrm{dd}, J=7.9,1.3 \mathrm{~Hz}, 1 \mathrm{H})$, 7.61 (td, $J=7.7,1.4 \mathrm{~Hz}, 1 \mathrm{H}), 7.44(\mathrm{td}, J=7.9,1.1 \mathrm{~Hz}, 1 \mathrm{H})$, 7.34 (d, $J=7.9 \mathrm{~Hz}, 1 \mathrm{H}), 3.61$ (d, $J=17.2 \mathrm{~Hz}, 1 \mathrm{H}), 3.44$ (s, $3 \mathrm{H}), 3.43$ (s, 3H), 3.08 (d, $J=17.2 \mathrm{~Hz}, 1 \mathrm{H}), 1.56(\mathrm{~s}, 3 \mathrm{H})$; ${ }^{13} \mathrm{C}$ NMR (125 MHz, $\left.\mathrm{CDCl}_{3}\right) \delta: 176.3,170.5,164.4,142.8$, 133.9, 129.3, 127.5, 124.9, 124.1, 51.8, 44.9, 44.4, 30.6, 27.3. HRMS (ESI) calcd for $\mathrm{C}_{14} \mathrm{H}_{16} \mathrm{NO}_{4}\left(\mathrm{M}+\mathrm{H}^{+}\right)$ 262.1079, found 262.1076.

2-(4-甲基-2-芐基-1,3-二酮)-1,2,3,4-四氢异喹啉-4乙酸甲酯(4): 淡黄色液体, 产率 $50 \% .{ }^{1} \mathrm{H}$ NMR (500 $\left.\mathrm{MHz}, \mathrm{CDCl}_{3}\right) \delta: 8.27$ (dd, $\left.J=7.9,1.1 \mathrm{~Hz}, 1 \mathrm{H}\right), 7.61$ (td, $J=7.8,1.4 \mathrm{~Hz}, 1 \mathrm{H}$ ), 7.43 (t, $J=7.2 \mathrm{~Hz}, 3 \mathrm{H}$ ), $7.35 \sim 7.27$ (m, 3H), $7.22(\mathrm{t}, J=7.3 \mathrm{~Hz}, 1 \mathrm{H}), 5.28 \sim 5.20(\mathrm{~m}, 2 \mathrm{H}), 3.63$ (d, $J=17.1 \mathrm{~Hz}, 1 \mathrm{H}), 3.33$ (s, 3H), 3.09 (d, $J=17.1 \mathrm{~Hz}$, 1H), 1.53 (s, 3H); ${ }^{13} \mathrm{C}$ NMR (125 MHz, $\left.\mathrm{CDCl}_{3}\right) \delta: 175.9$, $170.3,163.9,142.8,137.2,134.0,129.4,128.4,128.3$, 127.6, 127.3, 124.8, 124.1, 51.7, 45.2, 44.1, 43.8, 30.8. HRMS (ESI) calcd for $\mathrm{C}_{20} \mathrm{H}_{20} \mathrm{NO}_{4}\left(\mathrm{M}+\mathrm{H}^{+}\right)$338.1392, found 338.1388 .

2-(4-甲基-2-乙基-1,3-二酮)-1,2,3,4-四氢异喹啉-4乙酸甲酯(5): 无色液体, 产率 $23 \% .{ }^{1} \mathrm{H}$ NMR $(500 \mathrm{MHz}$, $\left.\mathrm{CDCl}_{3}\right) \delta: 8.27$ (dd, $\left.J=7.9,1.2 \mathrm{~Hz}, 1 \mathrm{H}\right), 7.61$ (td, $J=7.8$, $1.4 \mathrm{~Hz}, 1 \mathrm{H}$ ), 7.43 (td, $J=8.0,1.0 \mathrm{~Hz}, 1 \mathrm{H}), 7.34$ (d, $J=7.9$ $\mathrm{Hz}, 1 \mathrm{H}), 4.16 \sim 4.03(\mathrm{~m}, 2 \mathrm{H}), 3.61(\mathrm{~d}, J=17.1 \mathrm{~Hz}, 1 \mathrm{H})$, $3.43(\mathrm{~s}, 3 \mathrm{H}), 3.08$ (d, $J=17.1 \mathrm{~Hz}, 1 \mathrm{H}), 1.55$ (s, 3H), 1.24 (t, $J=7.1 \mathrm{~Hz}, 3 \mathrm{H}) ;{ }^{13} \mathrm{C} \mathrm{NMR}\left(125 \mathrm{MHz}, \mathrm{CDCl}_{3}\right) \delta: 175.7$, $170.4,163.8,142.8,133.8,129.2,127.5,124.9,124.1$, 51.7, 44.8, 44.3, 35.7, 30.6, 12.8. HRMS (ESI) calcd for $\mathrm{C}_{15} \mathrm{H}_{18} \mathrm{NO}_{4}\left(\mathrm{M}+\mathrm{H}^{+}\right)$276.1236, found 276.1231.

2-(4-甲基-2-苯基-1,3-二酮)-1,2,3,4-四氢异喹啉-4-
乙酸甲酯(6): 白色固体，产率 $42 \%$. m.p. $167 \sim 168{ }^{\circ} \mathrm{C}$; ${ }^{1} \mathrm{H}$ NMR $\left(500 \mathrm{MHz}, \mathrm{CDCl}_{3}\right) \delta: 8.30(\mathrm{dd}, J=7.9,1.3 \mathrm{~Hz}$, $1 \mathrm{H}), 7.67$ (td, $J=7.7,1.4 \mathrm{~Hz}, 1 \mathrm{H}), 7.53 \sim 7.47(\mathrm{~m}, 3 \mathrm{H})$, $7.47 \sim 7.39(\mathrm{~m}, 3 \mathrm{H}), 7.26(\mathrm{~s}, 1 \mathrm{H}), 3.62(\mathrm{~d}, J=17.2 \mathrm{~Hz}$, $1 \mathrm{H}), 3.50$ (s, 3H), 3.14 (d, $J=17.2 \mathrm{~Hz}, 1 \mathrm{H}), 1.68$ (s, 3H); ${ }^{13} \mathrm{C}$ NMR (125 MHz, $\left.\mathrm{CDCl}_{3}\right) \delta: 176.1,170.6,164.3,142.9$, $135.8,134.2,129.6,129.3,128.5,128.4,127.7,125.2$, 124.4, 51.9, 45.4, 44.9, 30.4. HRMS (ESI) calcd for $\mathrm{C}_{19} \mathrm{H}_{18} \mathrm{NO}_{4}\left(\mathrm{M}+\mathrm{H}^{+}\right)$324.1236, found 324.1232.

2-(2,4,6-三甲基-1,3-二酮)-1,2,3,4-四氢异喹啉-4-乙 酸甲酯(7): 白色固体, 产率 $67 \%$. m.p. $145 \sim 146{ }^{\circ} \mathrm{C} ;{ }^{1} \mathrm{H}$ NMR $\left(500 \mathrm{MHz}, \mathrm{CDCl}_{3}\right) \delta: 8.15(\mathrm{~d}, J=8.0 \mathrm{~Hz}, 1 \mathrm{H})$, $7.25 \sim 7.22(\mathrm{~m}, 1 \mathrm{H}), 7.12(\mathrm{~s}, 1 \mathrm{H}), 3.59(\mathrm{~d}, J=17.2 \mathrm{~Hz}$, 1H), 3.45 (s, 3H), 3.41 (s, 3H), 3.08 (d, $J=17.2 \mathrm{~Hz}, 1 \mathrm{H})$, 2.43 (s, 3H), 1.54 (s, 3H); ${ }^{13} \mathrm{C}$ NMR $\left(125 \mathrm{MHz}, \mathrm{CDCl}_{3}\right) \delta$ : 176.4, 170.6, 164.3, 144.7, 142.8, 129.3, 128.6, 124.6, $122.3,51.8,44.8,44.3,30.6,27.2,21.9$. HRMS (ESI) calcd for $\mathrm{C}_{15} \mathrm{H}_{18} \mathrm{NO}_{4}\left(\mathrm{M}+\mathrm{H}^{+}\right)$276.1236, found 276.1232.

2-(2,4-二甲基-6-乙氧基-1,3-二酮)-1,2,3,4-四氢异喹 啉-4-乙酸甲酯(8): 白色固体, 产率 63\%. m.p. 119 $120{ }^{\circ} \mathrm{C} ;{ }^{1} \mathrm{H}$ NMR $\left(500 \mathrm{MHz}, \mathrm{CDCl}_{3}\right) \delta: 8.21(\mathrm{~d}, J=8.8$ $\mathrm{Hz}, 1 \mathrm{H}), 6.93$ (dd, $J=8.8,2.4 \mathrm{~Hz}, 1 \mathrm{H}), 6.77$ (d, $J=2.3 \mathrm{~Hz}$, $1 \mathrm{H}), 4.11(\mathrm{q}, J=7.0 \mathrm{~Hz}, 2 \mathrm{H}), 3.60(\mathrm{~d}, J=17.2 \mathrm{~Hz}, 1 \mathrm{H})$, 3.47 (s, 3H), 3.40 (s, 3H), 3.03 (d, $J=17.2 \mathrm{~Hz}, 1 \mathrm{H}), 1.53$ (s, 3H), 1.45 (t, $J=7.0 \mathrm{~Hz}, 3 \mathrm{H}) ;{ }^{13} \mathrm{C}$ NMR $(125 \mathrm{MHz}$, $\left.\mathrm{CDCl}_{3}\right) \delta: 176.4,170.6,164.0,163.5,145.0,131.7,117.7$, $113.2,110.3,63.9,51.8,45.1,44.3,30.8,27.2,14.7$. HRMS (ESI) calcd for $\mathrm{C}_{16} \mathrm{H}_{20} \mathrm{NO}_{5}\left(\mathrm{M}+\mathrm{H}^{+}\right)$306.1341, found 306.1338 .

2-(2,4-二甲基-6-氟-1,3-二酮)-1,2,3,4-四氢异喹啉-4乙酸甲酯(9): 白色固体, 产率 $68 \%$. m.p. $161 \sim 162{ }^{\circ} \mathrm{C}$; ${ }^{1} \mathrm{H}$ NMR $\left(500 \mathrm{MHz}, \mathrm{CDCl}_{3}\right) \delta: 8.30(\mathrm{dd}, J=8.8,5.9 \mathrm{~Hz}$, $1 \mathrm{H}), 7.15 \sim 7.11(\mathrm{~m}, 1 \mathrm{H}), 7.01$ (dd, $J=9.3,2.4 \mathrm{~Hz}, 1 \mathrm{H})$, 3.62 (d, $J=17.4 \mathrm{~Hz}, 1 \mathrm{H}), 3.49$ (s, 3H), 3.41 (s, 3H), 3.01 (d, $J=17.4 \mathrm{~Hz}, 1 \mathrm{H}), 1.55$ (s, 3H); ${ }^{13} \mathrm{C}$ NMR $(125 \mathrm{MHz}$, $\left.\mathrm{CDCl}_{3}\right) \delta: 175.8,170.4,166.3(\mathrm{~d}, J=262.5 \mathrm{~Hz}), 163.4$, $145.85(\mathrm{~d}, J=8.7 \mathrm{~Hz}), 132.34(\mathrm{~d}, J=9.7 \mathrm{~Hz}), 121.42(\mathrm{~d}$, $J=2.5 \mathrm{~Hz}), 115.4(\mathrm{~d}, J=21.2 \mathrm{~Hz}), 111.2(\mathrm{~d}, J=22.5 \mathrm{~Hz})$, 51.9, $45.1(\mathrm{~d}, J=1.5 \mathrm{~Hz}), 44.2,30.5,27.4$. HRMS (ESI) calcd for $\mathrm{C}_{14} \mathrm{H}_{15} \mathrm{FNO}_{4}\left(\mathrm{M}+\mathrm{H}^{+}\right)$280.0985, found 280.0981 .

2-(2,4-二甲基-6-氯-1,3-二酮)-1,2,3,4-四氢异喹啉-4乙酸甲酯(10): 白色固体, 产率 $70 \%$. m.p. $172 \sim 173{ }^{\circ} \mathrm{C}$; ${ }^{1} \mathrm{H}$ NMR $\left(500 \mathrm{MHz}, \mathrm{CDCl}_{3}\right) \delta: 8.22(\mathrm{~d}, J=8.5 \mathrm{~Hz}, 1 \mathrm{H})$, $7.41(\mathrm{dd}, J=8.5,1.9 \mathrm{~Hz}, 1 \mathrm{H}), 7.31$ (d, $J=1.9 \mathrm{~Hz}, 1 \mathrm{H})$, 
3.61 (d, $J=17.4 \mathrm{~Hz}, 1 \mathrm{H}), 3.49$ (s, 3H), 3.41 (s, 3H), 3.04 (d, $J=17.4 \mathrm{~Hz}, 1 \mathrm{H}), 1.55$ (s, 3H); ${ }^{13} \mathrm{C}$ NMR $(125 \mathrm{MHz}$, $\mathrm{CDCl}_{3}$ ) $\delta: 175.7,170.4,163.5,144.6,140.4,130.9,128.2$, 124.5, 123.5, 51.9, 44.9, 44.2, 30.4, 27.4. HRMS (ESI) calcd for $\mathrm{C}_{14} \mathrm{H}_{15} \mathrm{ClNO}_{4}\left(\mathrm{M}+\mathrm{H}^{+}\right)$296.0690, found 296.0687.

2-(2,4-二甲基-6-澳-1,3-二酮)-1,2,3,4-四氢异喹啉-4乙酸甲酯(11): 白色固体, 产率 57\%. m.p. $156 \sim 157{ }^{\circ} \mathrm{C}$; ${ }^{1} \mathrm{H}$ NMR $\left(500 \mathrm{MHz}, \mathrm{CDCl}_{3}\right) \delta: 8.14(\mathrm{~d}, J=8.4 \mathrm{~Hz}, 1 \mathrm{H})$, $7.57(\mathrm{dd}, J=8.4,1.8 \mathrm{~Hz}, 1 \mathrm{H}), 7.47(\mathrm{~d}, J=1.8 \mathrm{~Hz}, 1 \mathrm{H})$, 3.60 (d, $J=17.4 \mathrm{~Hz}, 1 \mathrm{H}), 3.49$ (s, 3H), 3.41 (s, 3H), 3.03 (d, $J=17.4 \mathrm{~Hz}, 1 \mathrm{H}), 1.55$ (s, 3H); ${ }^{13} \mathrm{C}$ NMR $(125 \mathrm{MHz}$, $\left.\mathrm{CDCl}_{3}\right) \delta: 175.6,170.4,163.7,144.7,131.1,130.9,129.1$, 127.4, 123.9, 51.9, 44.9, 44.2, 30.4, 27.4. HRMS (ESI) calcd for $\mathrm{C}_{14} \mathrm{H}_{15} \mathrm{BrNO}_{4}\left(\mathrm{M}+\mathrm{H}^{+}\right) \quad 340.0184$, found 340.0180 .

2-(2,4-二甲基-6-三氟甲基-1,3-二酮)-1,2,3,4-四氢异 喹啉-4-乙酸甲酯(12): 白色固体, 产率 61\%. m.p. 142 $143{ }^{\circ} \mathrm{C} ;{ }^{1} \mathrm{H}$ NMR $\left(500 \mathrm{MHz}, \mathrm{CDCl}_{3}\right) \delta: 8.42(\mathrm{~d}, J=8.2$ $\mathrm{Hz}, 1 \mathrm{H}), 7.70$ (dd, $J=8.2,0.8 \mathrm{~Hz}, 1 \mathrm{H}), 7.57(\mathrm{~s}, 1 \mathrm{H}), 3.65$ (d, $J=17.5 \mathrm{~Hz}, 1 \mathrm{H}), 3.48(\mathrm{~s}, 3 \mathrm{H}), 3.44(\mathrm{~s}, 3 \mathrm{H}), 3.11$ (d, $J=17.5 \mathrm{~Hz}, 1 \mathrm{H}), 1.59(\mathrm{~s}, 3 \mathrm{H}) ;{ }^{13} \mathrm{C}$ NMR $(125 \mathrm{MHz}$, $\left.\mathrm{CDCl}_{3}\right) \delta: 175.5,170.4,163.3,143.7,135.4$ (q, $J=32.5$ $\mathrm{Hz}), 130.1,124.4$ (q, $J=3.8 \mathrm{~Hz}), 123.4$ (q, $J=267 \mathrm{~Hz})$, 121.3 (q, $J=3.7 \mathrm{~Hz}$ ), 120.1, 51.9, 45.0, 44.3, 30.4, 27.6. HRMS (ESI) calcd for $\mathrm{C}_{15} \mathrm{H}_{15} \mathrm{~F}_{3} \mathrm{NO}_{4}\left(\mathrm{M}+\mathrm{H}^{+}\right) 330.0953$, found 330.0949 .

2,4-二甲基-4-(2-二甲氧基-2-乙氧基)-1,3-二酮1,2,3,4-四氢异喹啉-6-乙酸甲酯(13): 白色固体, 产率 59\%. m.p. $154 \sim 155{ }^{\circ} \mathrm{C} ;{ }^{1} \mathrm{H}$ NMR (500 MHz, $\left.\mathrm{CDCl}_{3}\right) \delta$ : $8.35(\mathrm{~d}, J=8.2 \mathrm{~Hz}, 1 \mathrm{H}), 8.07$ (dd, $J=8.2,1.5 \mathrm{~Hz}, 1 \mathrm{H})$, $8.02(\mathrm{~d}, J=1.4 \mathrm{~Hz}, 1 \mathrm{H}), 3.97$ (s, $3 \mathrm{H}), 3.62(\mathrm{~d}, J=17.4 \mathrm{~Hz}$, $1 \mathrm{H}), 3.46$ (s, 3H), 3.43 (s, 3H), 3.17 (d, $J=17.4 \mathrm{~Hz}, 1 \mathrm{H})$, $1.58(\mathrm{~s}, 3 \mathrm{H}) ;{ }^{13} \mathrm{C} \mathrm{NMR}\left(125 \mathrm{MHz}, \mathrm{CDCl}_{3}\right) \delta: 175.9,170.6$, $165.9,163.6,143.0,134.8,129.5,128.3,128.2,125.6$, 52.6, 51.9, 44.9, 44.3, 30.3, 27.4. HRMS (ESI) calcd for $\mathrm{C}_{16} \mathrm{H}_{18} \mathrm{NO}_{6}\left(\mathrm{M}+\mathrm{H}^{+}\right)$320.1134, found 320.1130 .

2-(2,4-二甲基-6-叔丁基-1,3-二酮)-1,2,3,4-四氢异喹 啉-4-乙酸甲酯(14): 白色固体, 产率 60\%. m.p. 125 $126{ }^{\circ} \mathrm{C} ;{ }^{1} \mathrm{H}$ NMR $\left(500 \mathrm{MHz}, \mathrm{CDCl}_{3}\right) \delta: 8.18(\mathrm{~d}, J=8.3$ $\mathrm{Hz}, 1 \mathrm{H}), 7.46(\mathrm{dd}, J=8.3,1.8 \mathrm{~Hz}, 1 \mathrm{H}), 7.31(\mathrm{~d}, J=1.7 \mathrm{~Hz}$, 1H), 3.62 (d, $J=16.9 \mathrm{~Hz}, 1 \mathrm{H}), 3.43$ (s, 3H), 3.41 (s, 3H), 3.09 (d, $J=16.9 \mathrm{~Hz}, 1 \mathrm{H}), 1.56$ (s, 3H), 1.34 (s, 9H); ${ }^{13} \mathrm{C}$ NMR $\left(125 \mathrm{MHz}, \mathrm{CDCl}_{3}\right) \delta: 176.5,170.5,164.2,157.7$, 142.4, 129.1, 124.9, 122.3, 120.8, 51.7, 45.3, 44.4, 35.3,
31.1, 30.8, 27.2. HRMS (ESI) calcd for $\mathrm{C}_{18} \mathrm{H}_{24} \mathrm{NO}_{4}(\mathrm{M}+$ $\mathrm{H}^{+}$) 318.1705, found 318.1701 .

2-(2,4-二甲基-7-硝基-1,3-二酮)-1,2,3,4-四氢异喹 啉-4-乙酸甲酯(15): 淡黄色固体, 产率 67\%. m.p. 146 $147{ }^{\circ} \mathrm{C} ;{ }^{1} \mathrm{H}$ NMR $\left(500 \mathrm{MHz}, \mathrm{CDCl}_{3}\right) \delta: 9.10$ (d, $J=2.5$ $\mathrm{Hz}, 1 \mathrm{H}), 8.44(\mathrm{dd}, J=8.6,2.5 \mathrm{~Hz}, 1 \mathrm{H}), 7.56(\mathrm{~d}, J=8.6 \mathrm{~Hz}$, $1 \mathrm{H}), 3.70$ (d, $J=17.6 \mathrm{~Hz}, 1 \mathrm{H}), 3.49$ (s, $3 \mathrm{H}), 3.45$ (s, $3 \mathrm{H})$, $3.14(\mathrm{~d}, J=17.6 \mathrm{~Hz}, 1 \mathrm{H}), 1.61(\mathrm{~s}, 3 \mathrm{H}) ;{ }^{13} \mathrm{C}$ NMR $(125$ $\left.\mathrm{MHz}, \mathrm{CDCl}_{3}\right) \delta: 174.9,170.4,162.4,149.2,147.3,127.9$, 126.6, 125.9, 124.6, 52.1, 45.3, 44.1, 30.1, 27.7. HRMS (ESI) calcd for $\mathrm{C}_{14} \mathrm{H}_{15} \mathrm{~N}_{2} \mathrm{O}_{6}\left(\mathrm{M}+\mathrm{H}^{+}\right)$307.0930, found 307.0926 .

2-(2,4,7-三甲基-1,3-二酮)-1,2,3,4-四氢异喹啉-4-乙 酸甲酯(16): 白色固体, 产率 55\%. m.p. 124 125 ${ }^{\circ} \mathrm{C} ;{ }^{1} \mathrm{H}$ NMR (500 MHz, $\left.\mathrm{CDCl}_{3}\right) \delta: 8.07$ (s, 1H), 7.42 (dd, $J=7.9$, $1.1 \mathrm{~Hz}, 1 \mathrm{H}), 7.23(\mathrm{~d}, J=8.0 \mathrm{~Hz}, 1 \mathrm{H}), 3.58(\mathrm{~d}, J=17.1 \mathrm{~Hz}$, $1 \mathrm{H}), 3.44$ (s, 3H), 3.41 (s, 3H), 3.06 (d, $J=17.1 \mathrm{~Hz}, 1 \mathrm{H})$, $2.41(\mathrm{~s}, 3 \mathrm{H}), 1.53(\mathrm{~s}, 3 \mathrm{H}) ;{ }^{13} \mathrm{C}$ NMR $\left(125 \mathrm{MHz}, \mathrm{CDCl}_{3}\right) \delta$ : $176.5,170.6,164.5,139.9,137.4,134.9,129.4,124.7$, $124.1,51.8,44.7,44.4,30.6,27.3,20.9$. HRMS (ESI) calcd for $\mathrm{C}_{15} \mathrm{H}_{18} \mathrm{NO}_{4}\left(\mathrm{M}+\mathrm{H}^{+}\right)$276.1236, found 276.1232.

2-(2,4-二甲基-1,3-二酮-7-碘)-1,2,3,4-四氢异喹啉-4乙酸甲酯(17): 淡黄色体, 产率 $39 \% .{ }^{1} \mathrm{H}$ NMR (500 $\left.\mathrm{MHz}, \mathrm{CDCl}_{3}\right) \delta: 8.59(\mathrm{~d}, J=1.9 \mathrm{~Hz}, 1 \mathrm{H}), 7.91(\mathrm{dd}, J=8.3$, $1.9 \mathrm{~Hz}, 1 \mathrm{H}), 7.07$ (d, $J=8.3 \mathrm{~Hz}, 1 \mathrm{H}), 3.59$ (d, $J=17.3 \mathrm{~Hz}$, $1 \mathrm{H}), 3.47$ (s, 3H), 3.41 (s, 3H), 3.03 (d, $J=17.3 \mathrm{~Hz}, 1 \mathrm{H})$, 1.53 (s, $3 \mathrm{H}) ;{ }^{13} \mathrm{C}$ NMR $\left(125 \mathrm{MHz}, \mathrm{CDCl}_{3}\right) \delta: 175.7,170.4$, 162.9, 142.6, 142.2, 138.0, 126.6, 126.0, 92.5, 51.9, 44.8, 44.2, 30.4, 27.5. HRMS (ESI) calcd for $\mathrm{C}_{14} \mathrm{H}_{15} \mathrm{INO}_{4}(\mathrm{M}+$ $\left.\mathrm{H}^{+}\right) 388.0046$, found 388.0042 .

2-(2,4,8-三甲基-1,3-二酮)-1,2,3,4-四氢异喹啉-4-乙 酸甲酯(18): 白色固体, 产率 47\%. m.p. 91 92 ${ }^{\circ} \mathrm{C} ;{ }^{1} \mathrm{H}$ NMR (500 MHz, $\left.\mathrm{CDCl}_{3}\right) \delta: 7.45(\mathrm{t}, J=7.7 \mathrm{~Hz}, 1 \mathrm{H})$, $7.24 \sim 7.19(\mathrm{~m}, 2 \mathrm{H}), 3.63(\mathrm{~d}, J=17.3 \mathrm{~Hz}, 1 \mathrm{H}), 3.46(\mathrm{~s}$, $3 \mathrm{H}), 3.40$ (s, 3H), 3.08 (d, $J=17.2 \mathrm{~Hz}, 1 \mathrm{H}), 2.79$ (s, 3H), 1.54 (s, 3H); ${ }^{13} \mathrm{C}$ NMR $\left(125 \mathrm{MHz}, \mathrm{CDCl}_{3}\right) \delta: 175.9,170.7$, 164.9, 144.1, 142.9, 132.7, 131.6, 123.2, 122.2, 51.8, 45.0, 44.3, 31.2, 27.3, 24.0. HRMS (ESI) calcd for $\mathrm{C}_{15} \mathrm{H}_{18} \mathrm{NO}_{4}$ $\left(\mathrm{M}+\mathrm{H}^{+}\right)$276.1236, found 276.1232.

2-(2,4-二甲基-1,3-二酮-8-碘)-1,2,3,4-四氢异喹啉-4乙酸甲酯(19): 白色固体, 产率 $35 \%$. m.p. $156 \sim 157{ }^{\circ} \mathrm{C}$; ${ }^{1} \mathrm{H}$ NMR $\left(500 \mathrm{MHz}, \mathrm{CDCl}_{3}\right) \delta: 8.15(\mathrm{~d}, J=7.8 \mathrm{~Hz}, 1 \mathrm{H})$, 7.36 (d, $J=7.8 \mathrm{~Hz}, 1 \mathrm{H}), 7.19$ (t, $J=7.8 \mathrm{~Hz}, 1 \mathrm{H}), 3.63$ (d, $J=17.4 \mathrm{~Hz}, 1 \mathrm{H}), 3.48$ (s, 3H), 3.42 (s, 3H), 3.06 (d, $J=$ $17.4 \mathrm{~Hz}, 1 \mathrm{H}), 1.54$ (s, 3H); ${ }^{13} \mathrm{C} \mathrm{NMR}\left(125 \mathrm{MHz}, \mathrm{CDCl}_{3}\right) \delta$ : 
$174.7,170.4,162.1,145.6,142.8,133.7,124.6,124.5$, 95.7, 51.9, 45.7, 44.1, 30.9, 28.1. HRMS (ESI) calcd for $\mathrm{C}_{14} \mathrm{H}_{15} \mathrm{INO}_{4}\left(\mathrm{M}+\mathrm{H}^{+}\right) 388.0046$, found 388.0042 .

2-(2,4-二甲基-6,7-二甲氧基-1,3-二酮)-1,2,3,4-四氢 异喹啉-4-乙酸甲酯(20): 白色固体, 产率 44\%. m.p. 139 $140{ }^{\circ} \mathrm{C} ;{ }^{1} \mathrm{H}$ NMR (500 MHz, $\left.\mathrm{CDCl}_{3}\right) \delta: 7.70$ (s, 1H), $6.71(\mathrm{~s}, 1 \mathrm{H}), 3.96(\mathrm{~s}, 6 \mathrm{H}), 3.59$ (d, $J=17.0 \mathrm{~Hz}, 1 \mathrm{H}), 3.46$ (s, 3H), 3.41 (s, 3H), 3.01 (d, J=17.0 Hz, 1H), 1.55 (s, $3 \mathrm{H}) ;{ }^{13} \mathrm{C} \mathrm{NMR}\left(125 \mathrm{MHz}, \mathrm{CDCl}_{3}\right) \delta: 176.5,170.4,164.0$, 154.0, 148.6, 136.7, 117.9, 110.4, 106.1, 56.2, 56.1, 51.8, 44.9, 44.6, 30.6, 27.3. HRMS (ESI) calcd for $\mathrm{C}_{16} \mathrm{H}_{20} \mathrm{INO}_{6}$ $\left(\mathrm{M}+\mathrm{H}^{+}\right)$322.1291, found 322.1288.

2-(2,4-二甲基-5,7-二甲氧基-1,3-二酮)-1,2,3,4-四氢 异喹啉-4-乙酸甲酯(21): 淡黄色固体, 产率 48\%. m.p. $115 \sim 116{ }^{\circ} \mathrm{C} ;{ }^{1} \mathrm{H}$ NMR (500 MHz, $\left.\mathrm{CDCl}_{3}\right) \delta: 7.44$ (d, $J=$ $2.5 \mathrm{~Hz}, 1 \mathrm{H}), 6.70(\mathrm{~d}, J=2.4 \mathrm{~Hz}, 1 \mathrm{H}), 3.89 \sim 3.86(\mathrm{~m}, 6 \mathrm{H})$, 3.77 (d, $J=17.1 \mathrm{~Hz}, 1 \mathrm{H}), 3.43$ (s, 3H), 3.41 (s, 3H), 3.38 $(\mathrm{d}, J=17.3 \mathrm{~Hz}, 1 \mathrm{H}), 1.63(\mathrm{~s}, 3 \mathrm{H}) ;{ }^{13} \mathrm{C}$ NMR $(125 \mathrm{MHz}$, $\left.\mathrm{CDCl}_{3}\right) \delta: 177.4,171.7,164.5,159.6,157.4,127.3,122.8$, 105.3, 103.2, 55.7, 55.6, 51.5, 44.3, 42.2, 27.5, 26.1. HRMS (ESI) calcd for $\mathrm{C}_{16} \mathrm{H}_{20} \mathrm{NO}_{6}\left(\mathrm{M}+\mathrm{H}^{+}\right) 322.1291$, found 322.1287 .

2-(2,4-二甲基-1,3-二酮)-1,2,3,4-四氢异喹啉-4-乙酸 乙酯(22); 无色液体, 产率 51\%. ${ }^{1} \mathrm{H}$ NMR (500 MHz, $\left.\mathrm{CDCl}_{3}\right) \delta: 8.27(\mathrm{dd}, J=7.9,1.2 \mathrm{~Hz}, 1 \mathrm{H}), 7.62(\mathrm{td}, J=7.7$, $1.4 \mathrm{~Hz}, 1 \mathrm{H}), 7.44$ (td, $J=7.9,1.0 \mathrm{~Hz}, 1 \mathrm{H}), 7.36$ (d, $J=7.9$ $\mathrm{Hz}, 1 \mathrm{H}), 3.89 \sim 3.81(\mathrm{~m}, 2 \mathrm{H}), 3.61(\mathrm{~d}, J=17.0 \mathrm{~Hz}, 1 \mathrm{H})$, 3.42 (s, 3H), 3.05 (d, $J=17.0 \mathrm{~Hz}, 1 \mathrm{H}), 1.56$ (s, 3H), 0.98 $(\mathrm{t}, J=7.1 \mathrm{~Hz}, 3 \mathrm{H}) ;{ }^{13} \mathrm{C} \mathrm{NMR}\left(125 \mathrm{MHz}, \mathrm{CDCl}_{3}\right) \delta: 176.3$, 169.9, 164.4, 142.8, 133.9, 129.2, 127.5 124.9, 124.3, 60.7, 44.9, 44.8, 30.6, 27.3, 13.8. HRMS (ESI) calcd for $\mathrm{C}_{15} \mathrm{H}_{18} \mathrm{NO}_{4}\left(\mathrm{M}+\mathrm{H}^{+}\right)$276.1236, found 276.1232.

辅助材料(Supporting Information) 所有新化合物的 ${ }^{1} \mathrm{H}$ NMR 和 ${ }^{13} \mathrm{C} \mathrm{NMR}$ 谱图. 这些材料可以免费从本刊 网站(http://sioc-journal.cn/)上下载.

\section{References}

[1] (a) Ratnayake, R.; Lacey, E.; Tennant, S.; Gill, J. H.; Capon, R. J.
Chem. Eur. J. 2007, 13, 1610.

(b) Ratnayake, R.; Lacey, E.; Tennant, S.; Gill, J. H.; Capon, R. J. Org. Lett. 2006, 8, 5267.

(c) Zhang, A. H.; Jiang, N.; Gu, W.; Ma, J.; Wang, Y. R.; Song, Y. C.; Tan, R. X. Chem. Eur. J. 2010, 16, 14479.

(d) Pandey, R. C.; Toussaint, M. W.; Stroshane, R. M.; Kalita, C. C.; Aszalos, A. A.; Garretson, A. L.; Wei, T. T.; Byrne, K. M.; Geoghegan Jr., R. F.; White, R. J. J. Antibiot. 1981, 34, 1389.

(e) Pettit, G. R.; Gaddamidi, V.; Cragg, G. M.; Herald, D. L.; Sagawa, Y. Chem. Commun. 1984, 1693.

[2] (a) Pin, F.; Comesse, S.; Sanselme, M.; Darich, A. J. Org. Chem. 2008, 73, 1975.

(b) Mayer, S. C.; Banker, A. L.; Boschelli, F.; Di, L.; Johnson, M.; Kenny, C. H.; Krishnamurthy, G.; Kutterer, K.; Moy, F.; Petusky, S.; Ravi, M.; Tkach, D.; Tsou, H. R.; Xu, W. Bioorg. Med. Chem. Lett. 2008, 18, 3641.

[3] (a) Billamboz, M.; Bailly, F.; Lion, C.; Touati, N.; Vezin, H.; Calmels, C.; Andre'ola, M. L.; Christ, F.; Debyser, Z.; Cotelle, P. J. Med. Chem. 2011, 54, 1812.

(b) Billamboz, M.; Bailly, F.; Barreca, M. L.; De Luca, L.; Mouscadet, J. F.; Calmels, C.; Andre'ola, M. L.; Witvrouw, M.; Christ, F.; Debyser, Z.; Cotelle, P. J. Med. Chem. 2008, 51, 7717.

[4] Sire, B. Q.; Zard, S. Z. Chem. Commun. 2002, 2306.

[5] Li, L.; Zhao, Y. L.; Wang, H.; Li, Y. J.; Xu, X. X.; Liu, Q. Chem. Commun. 2014, 50, 6458.

[6] (a) Renaud, P.; Sibi, M. P. Radicals in Organic Synthesis, Wiley-VCH, Weinheim, 2001.

(b) Crich, D.; Brebion, F.; Suk, D. H. Topics in Current Chemistry, Vol. 263, Eds.: Gansauer, A., Springer, Berlin, 2006, pp. 1 38.

(c) Quiclet-Sire, B.; Zard, S. Z. Topics in Current Chemistry, Vol. 264, Eds.: Gansauer, A., Springer, Berlin, 2006, pp. 201 236.

[7] For reviews, see: (a) Wolfe, J. P. Eur. J. Org. Chem., 2007, 571. (b) Wolfe, J. P. Synlett 2008, 2913.

(c) Schultz, D. M.; Wolfe, J. P. Synthesis 2012, 351.

(d) Mai, W. P.; Wang, J. T.; Yang, L. R.; Yuan, J. W.; Mao, P.; Xiao, Y. M.; Qu, L. B. Chin. J. Org. Chem. 2014, 34, 1958 (in Chinese). (买文鹏, 王继涛, 杨亮茹, 袁金伟, 毛璞, 肖咏梅, 屈凌波, 有 机化学, 2014, 34, 1958.)

[8] (a) Li, X. Q.; Xu, X. S.; Hu, P. Z.; Xiao, X. Q.; Zhou, C. J. Org. Chem. 2013, 78, 7343.

(b) Li, X. Q.; Xu, X. S.; Tang, Y. C. Org. Biomol. Chem. 2013, 11, 1739.

(c) Li, X. Q.; Xu, X. S.; Zhou, C. Chem. Commun. 2012, 48, 12240. (d) Xu, X. S.; Tang, Y. C.; Li, X. Q.; Hong, G.; Fang, M. W.; Du, X. H. J. Org. Chem. 2014, 79, 446.

[9] (a) Kong, W. Q.; Casimiro, M.; Fuentes, N.; Merino, E. B.; Nevado, C. Angew. Chem., Int. Ed. 2013, 52, 13086.

(b) Li, L.; Deng, M.; Zheng, S. C.; Xiong, Y. P.; Tan, B.; Liu, X. Y. Org. Lett. 2014, 16, 504.

(c) Zhao, W. N.; Xie, P.; Zhang, M.; Niu, B.; Bian, Z. G.; Pittman, Jr. C.; Zhou, A. H. Org. Biomol. Chem. 2014, 12, 7690.

(Li, L.; Lu, Z.) 